\title{
Logarithmic relaxation due to minimization of interactions in the Burridge-Knopoff model
}

\author{
B. A. H. Huisman ${ }^{1}$ and A. Fasolino ${ }^{1,2}$ \\ ${ }^{1}$ Van 't Hoff Institute for Molecular Sciences, University of Amsterdam, Nieuwe Achtergracht 166, 1018 WV Amsterdam, The Netherlands \\ ${ }^{2}$ Theory of Condensed Matter, Institute for Molecules and Materials, Radboud University Nijmegen, Toernooiveld 1, 6525 ED \\ Nijmegen, The Netherlands \\ (Received 13 January 2006; published 10 August 2006)
}

\begin{abstract}
The time evolution of macroscopic quantities describing the relaxation of complex systems often contains a domain with logarithmic time dependence. This logarithmic behavior at the macroscopic level is often associated with strongly interacting elements at the microscopic level, whose interactions depend significantly on their history. In this paper we show that stress relaxation in the Burridge-Knopoff (BK) model of multicontact friction behaves logarithmically, when the model is in, or close to, the solitary state where the elements move independently. For this regime we present an automaton that allows us to follow the decay of stress relaxation over the entire range where it behaves logarithmically in time. We show that our model can be mapped onto a system of noninteracting elements subject to a uniform distribution of forces, for which logarithmic stress relaxation is derived analytically.
\end{abstract}

DOI: 10.1103/PhysRevE.74.026110

PACS number(s): 81.40.Pq, 62.40.+i, 68.35.Af, 05.40.-a

\section{INTRODUCTION}

The gradual return of complex systems, such as spin glasses [1], structural glasses [2], granular materials [3,4], proteins [5], and microgel pastes [6], towards thermodynamic equilibrium is often anomalously slow. Macroscopic quantities describing this relaxation frequently exhibit a range of times where their time dependence is logarithmic. When subjected to a constant weak driving force, the response of these complex systems, including biological materials, decays logarithmically in time as well [7-9]. Both types of slow behavior are usually associated with thermally activated processes limiting the rearrangement of the microscopic constituents. The behavior can arise either from the parallel relaxation of independent elements, or by a sequential series of correlated complex events [10]. When modeling their behavior, the constituents are often [10-12], described as elements $i$ having an energy $\epsilon_{i}$ in their microscopic state. The elements are separated from a more favorable state with energy $\epsilon_{i}^{\prime}<\epsilon_{i}$ by an energy barrier $\Delta E_{i}$, which they can overcome thermally. Energies $\epsilon_{i}, \Delta E_{i}$, or $\epsilon_{i}^{\prime}$ can all depend on the state of the surrounding elements $\epsilon_{j \neq i}$, and it is by these couplings that complex interactions are introduced into the models.

The exhaustion model is an example of a system displaying logarithmic relaxation without complexity [12]. It has noninteracting elements distributed in such a way that all values of the energy barriers are equally likely. The occurrence of such a distribution of energy barriers is regarded as very artificial.

The Burridge-Knopoff [13] (BK) model with viscous damping has been studied as an appropriate model for sliding friction in the boundary lubrication regime [14]. At finite temperature it displays logarithmic stress relaxation over a range of times [14], with a behavior similar to that found for models with hierarchically constrained dynamics [11]. We have shown that, when driven at a constant velocity, this version of the BK model reaches a solitary state where the model's elements move independently [15]. Here we will show that the BK model displays logarithmic relaxation when its elements behave almost independently. The solitary state displays strong analogies to the exhaustion model, with the difference that the force acting on the elements before they slip, and not the energy barriers, are all equally likely. By introducing an automaton model which is exact for the solitary state, we can follow the stress relaxation over an extended time span. The results can be interpreted by mapping the automaton onto a simpler system of noninteracting elements subjected to an almost uniform distribution of forces. Analytical results can be derived for this simpler system.

This paper is organized as follows. In Sec. II the dynamics of the BK model is reviewed. In Sec. III we examine the stress relaxation at finite temperatures (i) by full simulations, (ii) by an exact automaton model, and (iii) by approximate analytical derivations based on a simplified version of the automaton. A summary and conclusions are given in Sec. IV. Details of the analytical derivations are given in the Appendix.

\section{THE BK MODEL OF MULTICONTACT FRICTION}

The static friction force between two surfaces is defined as the amount of lateral force that has to be applied for the surfaces to start sliding. Experimentally it is often found to be approximately twice the dynamic friction force, the force needed to keep the surfaces in relative motion [16]. Both the static and dynamic friction force usually depend linearly on the force perpendicular to the contact surface, a finding called the Coulomb or Amonton law. Microscopically the real contact area between two surfaces is only a fraction of the macroscopic area of contact, either because of contaminants between the surfaces, or because of the roughness of the surfaces. The real contact area is approximately linear with the perpendicular load force, yielding the linear dependence of the friction forces on load [17]. As the surfaces slide, the force increases at the contact points. This increase continues until the static friction force of a contact is 


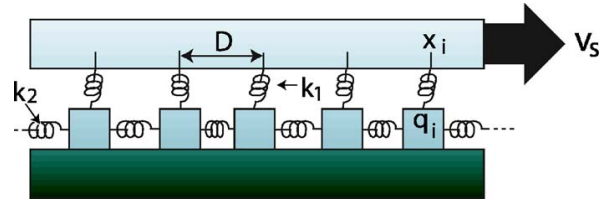

FIG. 1. (Color online) The Burridge Knopoff (BK) model. Blocks of mass $m$ are connected to neighboring blocks by springs of spring constant $k_{2}$ and to the top plate by springs of spring constant $k_{1}$. The top plate is moved at a low constant velocity $v_{s}$. Blocks move when they experience a force larger than the static friction force and stop again when their velocity with respect to the bottom plate vanishes.

reached, and the contact breaks or slips. A breaking or slipping contact releases the potential energy stored in it, and quickly new contacts are made. If the sliding velocity is low enough, only a tiny fraction of the contacts are in motion or in the process of breaking. In this case, the case of multicontact friction, the dynamic friction force is mostly due to the static friction force pinning the microscopic contacts.

The BK model (Fig. 1) is a model for this type of frictional systems. It was introduced to describe the dynamics of earthquake faults [13], but later adapted to describe multicontact friction [14]. Slowly the top plate moves at a constant velocity $\dot{x}=v_{s}$, and in doing so increases the force via springs of spring constant $k_{1}$ on $N$ blocks of mass $m$, representing the microscopic contacts. The blocks are connected to the top plate at fixed distances $D$. Their points of attachment on the top plate are denoted by $x_{i}=x+i D$, while their positions are expressed by $q_{i}$. All blocks are connected to their first neighbors by springs of spring constant $k_{2}$, representing the elastic interaction between the contacts. Furthermore, when in motion, the blocks are damped by a viscous force $-2 m \gamma \dot{q}_{i}$, where $\dot{q}_{i}$ is the velocity of block $i$ and $\gamma$ is the damping constant. The damping allows for the dissipation of energy as the contacts move. The total force experienced by a block is

$$
\begin{aligned}
\sigma_{i} & =k_{1}\left(x_{i}-q_{i}\right)+k_{2}\left(q_{i-1}-q_{i}\right)+k_{2}\left(q_{i+1}-q_{i}\right)-2 m \gamma \dot{q}_{i} \\
& =k_{1} x_{i}-\left(k_{1}+2 k_{2}\right) q_{i}+k_{2}\left(q_{i-1}+q_{i+1}\right)-2 m \gamma \dot{q}_{i} .
\end{aligned}
$$

The blocks are anchored to the bottom immobile plate by a static friction force of size $\sigma_{s}$. Due to the static friction, blocks start to move only when they experience a force $\sigma_{i}$ $\geqslant \sigma_{s}$, and stop once their velocity vanishes again, i.e., when $\dot{q}_{i}=0$. Whether a block is moving or not, is characterized by the dimensionless quantity $h_{i}$

$$
h_{i} \equiv \begin{cases}0, & \text { if } \dot{q}_{i}=0 \text { ( pinned) } \\ 1, & \text { otherwise (moving) } .\end{cases}
$$

For convenience we shift the positions of the blocks by $\Delta q_{i}=i D$, and scale them by $\sigma_{s} / k_{1}$. We scale time by $\sqrt{m / k_{1}}$ and forces by $\sigma_{s}$, so that Eq. (1) becomes dimensionless

$$
\sigma_{i}=x-\omega_{0}^{2} q_{i}+\tilde{k}_{2}\left(q_{i-1}+q_{i+1}\right)-2 \tilde{\gamma} \dot{q}_{i},
$$

where $\omega_{0}^{2}=1+2 \widetilde{k}_{2}, \tilde{k}_{2}=k_{2} / k_{1}$, and $\tilde{\gamma}=\gamma \sqrt{m / k_{1}}$. From now on we will only consider dimensionless quantities and will omit

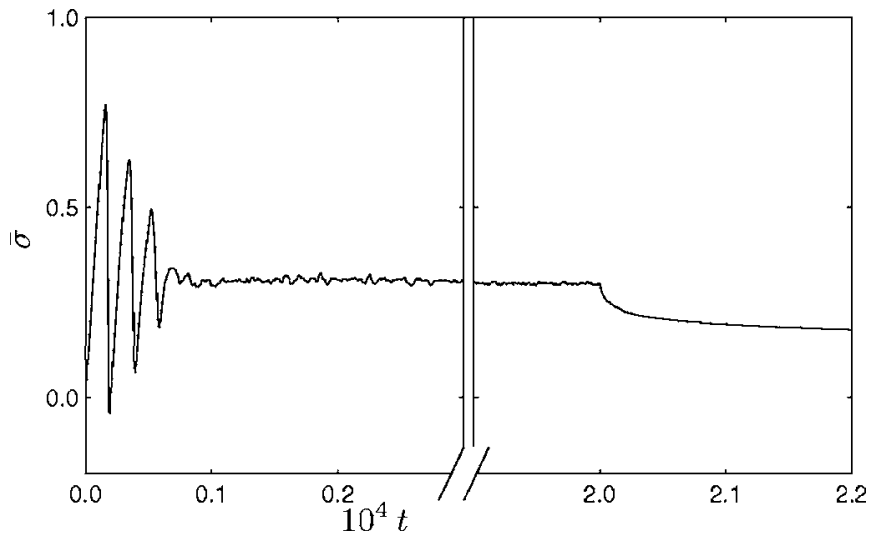

FIG. 2. Mean force $\bar{\sigma}$ as a function of time, for a system with $N=10000$ blocks, $k_{2}=1, \gamma=0.5$, driven at $v_{s}=0.005$ at a temperature $k_{b} T=\epsilon / 16.7$ [18]. The quantity $\epsilon$ is defined in Eq. (6). Notice the gap in time indicated by vertical lines. At $t=2 \times 10^{4}$ the top plate is stopped $\left(v_{s}=0\right)$, and the force relaxes approximately as a logarithm of time.

the tilde. Note that due to the normalization, the static friction force is $\sigma_{s}=1$. To simulate the motion of the blocks, we integrate their dimensionless equations of motion

$$
\ddot{q}_{i}=h_{i}\left[x-\omega_{0}^{2} q_{i}+k_{2}\left(q_{i+1}+q_{i-1}\right)-2 \gamma \dot{q}_{i}\right] \equiv h_{i} \sigma_{i},
$$

where

$$
h_{i}(t+d t)= \begin{cases}0, & \dot{q}_{i}(t) \dot{q}_{i}(t+d t)<0 \\ 1, & \sigma_{i}(t+d t) \geqslant 1 \\ h_{i}(t), & \text { otherwise }\end{cases}
$$

with $d t$ the time step of numerical integration. If $h_{i}(t+d t)$ $=0, \dot{q}_{i}(t+d t)$ is set to zero. We use a fourth-order RungeKutta algorithm with $d t=0.005$. The initial positions $q_{i}(0)$ are chosen from a uniform random distribution $q_{i}(0)$ $=[-0.005,0.005]$; furthermore, $x(0)=0$ and $\dot{q}_{i}(0)=0$. We use periodic boundary conditions. To describe realistic sliding systems, we choose $k_{2}=1$ and $\gamma=0.5$ as was suggested in Ref. [14]. Initially we drive the system at $v_{s}=0.005$. At this velocity the individual blocks move only a small fraction of the time.

In Fig. 2 the mean force $\bar{\sigma}$ is shown for a typical simulation. At $t=0$ we start driving the top plate at a velocity $v_{s}$, and at $t=20000$ we stop driving the top plate. Initially the blocks do not exert much force on their neighbors, as they are positioned at almost equal intervals along the plate; almost all the force acting on the blocks is due to the motion of the top plate and the force acting on all the blocks is approximately equal. As the top plate moves forward, $\sigma_{i}$ increases until, nearly simultaneously, all blocks start to move when $\sigma_{i}=1$. All blocks stop almost simultaneously as well. Such sudden movement of one or more blocks is called a slip event. Motion through such collective slip events is not stable for moderate values of $k_{2}$, and the distribution of forces $P(\sigma)$ widens progressively until at some point, at all times, approximately the same small fraction of blocks is moving while the others are at rest. This is illustrated in Fig. 2 , where the initial sawtooth behavior of the mean force $\bar{\sigma}$ 

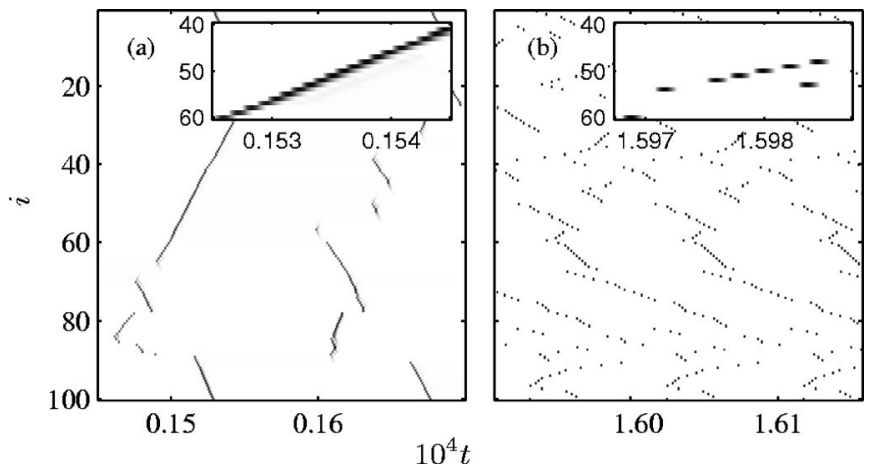

FIG. 3. Block velocity as a function of time $t$ [(a) short times, (b) long times] and block index $i$ in a system of $N=100$ blocks, $k_{2}=1, \gamma=0.5, T=0$, driven at $v_{s}=0.005$. Gray scale denotes velocity: when white, the blocks do not move, when dark, the blocks are in motion. Note that blocks move only a fraction of the time. Insets zoom in on part of the graph. (a) Initially blocks move in concert; slipping blocks drag their neighbors along. The horizontal black lines of blocks $i$ and $i+1$ overlap partially, resulting in wide diagonal lines in this plot. (b) Eventually the blocks are in the solitary state. Velocities of neighboring blocks do not overlap, although they are correlated. As a consequence, the diagonal lines appear as dashed. Note that in the solitary state (b) the profile is periodic in time, whereas the profile in (a) is not.

acting on the blocks settles in time roughly to a constant. The relaxation occurring when the driving plate is stopped is described in Sec. III.

In Ref. [15] we have shown that for $k_{2} \lesssim 1.6$ and $\gamma \approx 0.5$ the blocks evolve to solitary motion. In solitary motion a block $i$ moves while its first neighbors stay motionless throughout the motion of block $i$. In Fig. 3 the velocity profile of a driven BK model at $T=0$ is shown at short and long times to illustrate the difference between collective and solitary motion. At short times the motion of neighboring blocks overlaps as illustrated by the broad diagonal lines in the velocity profile. At long times in the solitary state, the motion of one block is always separated in time from both of its neighbors. There are still diagonal lines in the profile, but they appear as "dashed."

\section{STRESS RELAXATION AT FINITE TEMPERATURE}

At low enough driving velocities and high enough temperatures the contacts between two surfaces can break due to thermal fluctuations before they experience the static friction force. Blocks can start moving while $\sigma_{i}<1$. Following Persson $[14,15]$, the energy block $i$ needs to gain by thermal fluctuations before it can slip is given by

$$
\Delta E_{i}=\epsilon\left(1-\sigma_{i}^{2}\right) \quad \text { with } \epsilon=1 / 2 \omega_{0}^{2},
$$

where $\epsilon$ is the maximum size of the energy barrier. The probability $w_{i} d t$ that block $i$ slips (i.e., overcomes the energy barrier) within a time $d t$ is assumed to be

$$
w_{i} d t=\nu \exp \left(-\beta \Delta E_{i}\right) d t,
$$

where $\nu$ is an attempt frequency $\beta=1 / k_{B} T, T$ the temperature, and $k_{B}$ the Boltzmann constant. In practice, finite tem- perature is simulated by drawing a random number $r_{i}$ $=[0,1]$ at each integration step for each block, and if $r_{i}$ $<w_{i} d t$, where $d t$ is the step of integration, the static friction force is decreased to zero by setting $h_{i}=1$. In Ref. [15] we showed that the solitary state is stable for temperatures $k_{B} T$ $<\epsilon / 30$, and still dominates the dynamics at $k_{B} T<\epsilon / 15$.

After we stop driving a system that is in the solitary state, only thermal fluctuations can cause blocks to slip, even though the blocks that are moving at that time will finish their motion. Persson has found that relaxation of the average force is logarithmic over a range of times [14]. In Fig. 2 the relaxation of the mean force is shown, after the upper surface is stopped. The fact that the relaxation has a logarithmic part makes it especially hard to study with conventional simulations. While the relaxation slows down each order of time, the integration time step has to be kept constant. We can, however, make use of the knowledge of the solitary state to extend our analysis over many decades of time, allowing us to follow the logarithmic time behavior even for low temperatures.

In Ref. [15] we calculated the distance a block moves in the solitary state. We showed that a block experiencing a force $\sigma_{\text {init }}$ at the moment it starts to move, moves a distance

$$
\Delta q\left(\sigma_{\text {init }}\right)=\frac{\sigma_{\text {init }}}{\omega_{0}^{2}}\{1+\exp (-\gamma \pi / \omega)\} \equiv \sigma_{\text {init }} \Delta q_{\max }
$$

in a time

$$
\delta t=\frac{\pi}{\omega} \quad \text { with } \omega=\sqrt{\omega_{0}^{2}-\gamma^{2}}
$$

before its velocity vanishes and it sticks again. The force after the block has slipped is given by

$$
\sigma_{\text {after }}=\sigma_{\text {init }}-\omega_{0}^{2} \Delta q\left(\sigma_{\text {init }}\right)=\sigma_{\text {init }}\left(1-\omega_{0}^{2} \Delta q_{\text {max }}\right)
$$

and the slip of block $i$ increases the force on the neighboring blocks by $k_{2} \Delta q\left(\sigma_{\text {init }}\right)$. For $k_{2}=1, \gamma=0.5$, and $\sigma_{i}=1$ these quantities evaluate to $\Delta q_{\max } \approx 0.46, \quad \delta t \approx 1.9, \quad \sigma_{\text {after }}$ $\approx-0.38 \sigma_{\text {init }}$.

Since in the solitary state blocks do not move at the same time as their neighbors, we can use this knowledge to construct an automatonlike model to describe the relaxation of the mean force in the solitary state after the drive is removed. In this automaton model the blocks are represented only by the forces $\sigma_{i}$ acting on them. The motion of block $i$ is represented by an instantaneous decrease of the force acting on it, and an increase of the force acting on its neighbors

$$
\begin{gathered}
\sigma_{i-1} \rightarrow \sigma_{i-1}+k_{2} \sigma_{i} \Delta q_{\max }, \\
\sigma_{i+1} \rightarrow \sigma_{i+1}+k_{2} \sigma_{i} \Delta q_{\max }, \\
\sigma_{i} \rightarrow \sigma_{i}-\left(1+2 k_{2}\right) \sigma_{i} \Delta q_{\max } .
\end{gathered}
$$

With the drive removed, the motion of blocks in the solitary state is completely thermally activated. As for the full simulation at each automaton cycle, we take $N$ random numbers $r_{i}=[0,1]$, calculate $w_{i} d t$ from Eqs. (6) and (7), and apply the rule of Eq. (11) for each block with $r_{i}<w_{i} d t$. As the initial state for the automaton we use the forces $\sigma_{i}$ of a full simu- 

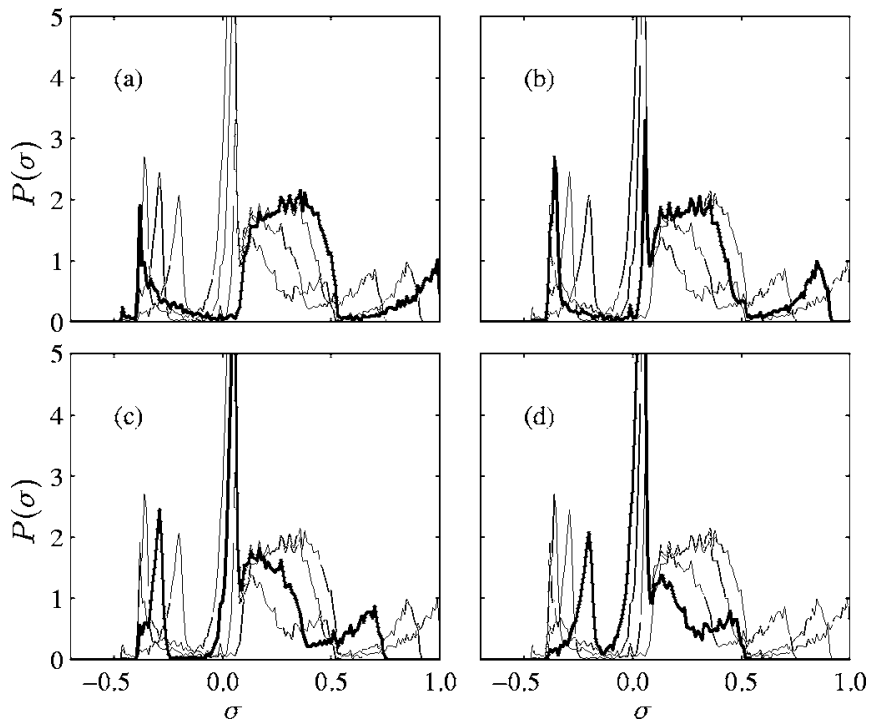

FIG. 4. The distribution of forces during relaxation after (a) $t$ $\approx 10^{1}$, (b) $t \approx 10^{6}$, (c) $t \approx 10^{14}$ and (d) $t \approx 10^{23}$, calculated by applying the automaton rules defined in Eq. (11) and the procedures as defined in the text. The thick black line denotes the distribution at the given time; the thin gray lines denote the distribution at the three other times. Note that the maximum force in the distribution decreases with increasing time and that the distribution becomes peaked around $\sigma=0 . N=10000, k_{2}=1, \gamma=0.5, k_{B} T=\epsilon / 66.7$.

lation at the time we remove the drive. However, since a fraction of the blocks is still in motion at the time the upper plate is stopped, we first complete the motion of these blocks at zero temperature before using the forces as an initial state of the automaton. We can tune the interval between consecutive automaton cycles so that we can choose the average number of blocks $n$ that slips at each cycle

$$
d t=\frac{n}{N \bar{w}} \Rightarrow \bar{w} d t=\frac{n}{N}, \quad \bar{w}=\frac{1}{N} \sum_{i}^{N} w_{i} .
$$

As the average force decreases, $\bar{w}$ decreases and the interval $d t$ increases. By increasing the time step accordingly, this method keeps the amount of blocks $n$ that move at each step approximately constant. This makes the computational cost of calculating the full relaxation curve by the automaton model temperature independent, and allows us to calculate exponential simulation time roughly in linear computer time. A smaller $n / N$ results in a smoother decay at a higher computational cost. The automaton is accurate on time scales larger than the typical duration of a slip event $\delta t$ of Eq. (9). The value of $n$ should be a small fraction of $N$ to provide good time and force resolution.

In Fig. 4 we show the distribution of forces at the moment the top plate is stopped and at various stages during the relaxation. The maximal force in the distribution decreases with increasing relaxation time. The consequent narrowing of the distribution is compensated by the increased probability around $\sigma=0$. This suggests that only blocks experiencing

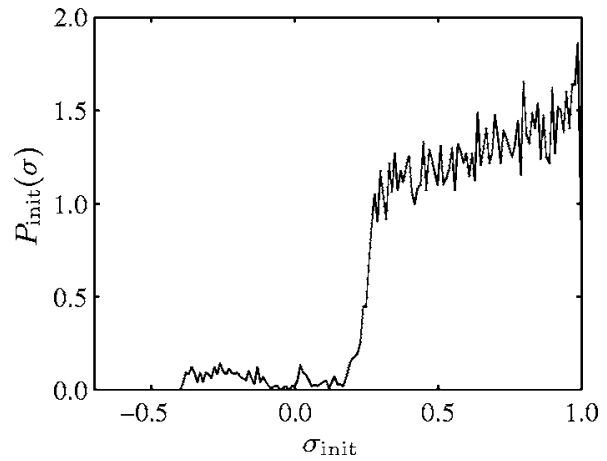

FIG. 5. The distribution $P_{\text {init }}(\sigma)$ of forces $\sigma_{\text {init }}$ acting on blocks just before they slip, for the first 10000 slip events in the simulation of Fig. 4. Note that this distribution resembles a uniform distribution, while the distribution in Fig. 4 has a highly peaked nature. $N=10000, k_{2}=1, \gamma=0.5, k_{B} T=\epsilon / 66.7$.

the maximal force in the system have a significant probability to slip. In the limit of extremely low temperature only blocks experiencing the maximal force have a non-negligible probability to slip as a consequence of the exponential behavior given in Eq. (7).

In Fig. 5 we show the distribution of the force $\sigma_{\text {init }}$ acting on each block just before a thermally excited slip occurs. It is created by measuring the stress acting on each block that slips for the first $N$ slip events. The distribution $P\left(\sigma_{\text {init }}\right)$ initially is peaked around $\sigma_{\text {init }}=1$ and progressively becomes more uniform, extending to lower stresses, as more blocks slip. It is much smoother than the one shown in Fig. 4 and is approximately uniform for $\sigma_{\text {init }} \gtrsim 0.25$.

As block $i$ slips, the force on its neighbors increases so that there is a continuous supply of blocks near the maximum force in the distribution. From Eq. (11) it is clear that the net result on the average force is a decrease of $\Delta \bar{\sigma}$ $=-\sigma_{i} \Delta q_{\max } / N$, proportional to the force $\sigma_{i, \text { init }}$ experienced by a block just before it slips. The fact that the $\sigma_{i, \text { init }}$ is rather uniform suggests a simplified description of the relaxation. Instead of advancing the automaton [Eq. (11)], we study the relaxation of the force on block $i$ only, resulting from a uniform distribution. This is a reasonable approximation because the distribution of Fig. 5 that we represent as uniform already accounts for the effect that slip of block $i$ has on its neighbors. This simplified automaton relaxes under the rule

$$
\sigma_{i, \text { init }} \rightarrow \sigma_{i, \text { init }}-\sigma_{i, \text { init }} \Delta q_{\max } .
$$

A further simplification allowing analytical results is to assume complete relaxation for each slip, namely,

$$
\sigma_{i, \text { init }} \rightarrow 0
$$

From Eq. (7) the probability $\pi_{i}$ that block $i$ has not yet slipped after a time $t$ is

$$
\pi_{i}(t)=\exp \left(-w_{i} t\right)=\exp \left\{-\nu t \exp \left[-\beta \epsilon\left(1-\sigma_{i, \text { init }}\right)\right]\right\} .
$$

The fraction of blocks $\bar{n}(t) / N$ that have not yet slipped after a time $t$ after the top plate was stopped is given by 


$$
\begin{aligned}
\frac{\bar{n}(t)}{N} & =\sum_{i=1}^{N} \pi_{i}(t) P_{\text {init }}\left(\sigma_{i, \text { init }}\right) \\
& =\sum_{i=1}^{N} P_{\text {init }}\left(\sigma_{i, \text { init }}\right) e^{-\nu t \exp \left(-\beta \epsilon\left[1-\sigma_{i, \text { init }}^{2}\right]\right)} .
\end{aligned}
$$

Consequently the average force $\bar{\sigma}(t)$ at time $t$ is given by

$$
\bar{\sigma}(t)=\sum_{i=1}^{N} \sigma_{i, \text { init }} P_{\text {init }}\left(\sigma_{i, \text { init }}\right) e^{-\nu t \exp \left(-\beta \epsilon\left[1-\sigma_{i, \text { init }}^{2}\right]\right)} .
$$

If we assume $N \gg 1$ we can safely replace the sum over $\sigma_{i \text {,init }}$ by an integral over $\sigma$,

$$
\bar{\sigma}(t)=\int_{-\infty}^{\infty} \sigma P_{\text {init }}(\sigma) e^{-\nu t \exp \left(-\beta \epsilon\left[1-\sigma^{2}\right]\right)} d \sigma,
$$

and integrate Eq. (19) numerically. We can either use the true form of $P_{\text {init }}(\sigma)$ given in Fig. 5, or we can approximate it by a uniform distribution of forces $P_{\text {init }}(\sigma)=1$ over $0 \leqslant \sigma_{i \text {,init }}$ $\leqslant 1$;

$$
\bar{\sigma}(t)=\int_{0}^{1} \sigma e^{-\nu t \exp \left(-\beta \epsilon\left[1-\sigma^{2}\right]\right)} d \sigma .
$$

We can write Eq. (17) in a similar fashion

$$
\frac{\bar{n}(t)}{N}=\int_{0}^{1} e^{-\nu t \exp \left(-\beta \epsilon\left[1-\sigma^{2}\right]\right)} d \sigma .
$$

Approximations of Eq. (20) for short, intermediate, and long times are derived in the Appendix. At short times the relaxation is given by

$$
\lim _{t \rightarrow 0} \bar{\sigma}(t)=\frac{1}{2}-\frac{1}{2 \beta \epsilon}\{[1-\exp (-\beta \epsilon)] \nu t\} .
$$

Since at low temperatures $\exp (-\beta \epsilon) \approx 0$, the force does not relax considerably at short times. For long times, the relaxation is proportional to

$$
\lim _{t \rightarrow \infty} \bar{\sigma}(t) \propto \exp [-\nu t \exp (-\beta \epsilon)]
$$

which gives an indication of how long it approximately takes for the system to fully relax, $t \approx \exp (\beta \epsilon) / \nu$.

The most interesting regime is at intermediate times, where the relaxation is logarithmic

$$
\bar{\sigma}(t) \approx \frac{1}{2}-\frac{1}{2 \beta \epsilon}[\hat{\gamma}+\log (\nu t)],
$$

here $\hat{\gamma}$ is the Euler-Mascheroni constant $\hat{\gamma} \approx 0.577$.

In Ref. [14] Persson derives Eq. (24), albeit without the additive constant $\hat{\gamma}$, by making two assumptions that we have not needed in our derivation. First, the assumption that the energy barrier can be expanded linearly in terms of $\xi$ $=1-\sigma$, and second, that the fraction of blocks $n / N$ that have not yet slipped is proportional to the mean force acting on the blocks. On the contrary, for very low temperatures, we can show that $\bar{\sigma} \propto \bar{n}^{2}$. At extremely low temperatures only the

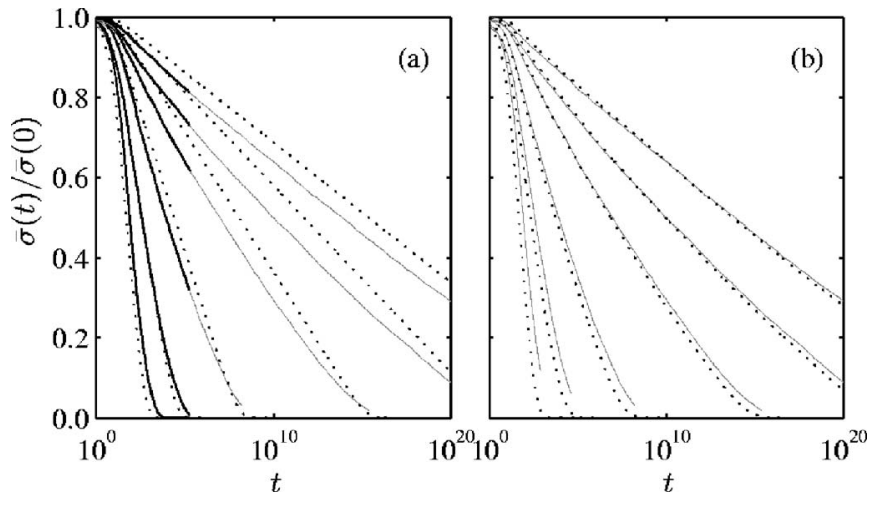

FIG. 6. Comparison of the different methods to calculate the stress relaxation for different temperatures. All initial configurations are taken after a $t=20000$ simulation with $k_{2}=1, \gamma=0.5, v_{s}$ $=0.005, N=10000$. All curves are scaled to $\bar{\sigma}(t=0)$. Curves from left to right for decreasing temperatures, $k_{B} T=\epsilon / 4.2, k_{B} T=\epsilon / 8.3$, $k_{B} T=\epsilon / 16.7, k_{B} T=\epsilon / 33.3, k_{B} T=\epsilon / 50, k_{B} T=\epsilon / 66.7$, respectively. (a) The thick black line up to $t=2 \times 10^{5}$ denotes the stress relaxation calculated by the full integration of the equations of motion. The thin gray line denotes the calculation of the slip of $2 N$ blocks, by the automaton of Eq. (11) started using the same configuration of forces as the full simulation. The dotted black line is the numerical integral of Eq. (20). (b) The thin gray line denotes the automaton calculation reported in (a). The dotted line is the numerical integral of Eq. (19), with the actual $P_{\text {init }}(\sigma)$ calculated over $2 N$ automaton slips (see Fig. 5).

block experiencing the maximal force has a non-negligible probability to slip. In this case we can assume that the blocks slip in order of decreasing stress, much like in the driven $T$ $=0$ case, but with exponentially increasing intervals between consequent block slips. Consider a system of $N$ forces $\sigma_{i}$ $=0,1 /(N-1), \ldots, 1$, so that $\sum_{i=1}^{N} \sigma_{i} / N=1 / 2$, relaxing under the rule of Eq. (14). The mean force at a time where $n$ blocks have not yet slipped is

$$
\bar{\sigma}(n)=\frac{1}{N} \sum_{i=1}^{n} \frac{(i-1)}{(N-1)}=\frac{n(n-1)}{2 N(N-1)} \approx \frac{n^{2}}{2 N^{2}} .
$$

By automaton simulations implementing Eq. (14), we found that Eq. (25) holds reasonably for temperatures $k_{B} T \lesssim \epsilon / 15$ and becomes nearly perfect at $k_{B} T \lesssim \epsilon / 60$.

In Fig. 6 we compare the different methods and approximations for calculating the stress relaxation $\bar{\sigma}(t)$. All curves are scaled by $\bar{\sigma}(t=0)$. While the results of the automaton calculations [Eq. (11)] are perfectly in agreement with the full simulations, the analytical approximation of Eq. (20) is not. This is due to the fact that $P_{\text {init }}(\sigma)$ is not a truly uniform distribution. If instead we integrate Eq. (19) using $P_{\text {init }}(\sigma)$, the agreement between the numerical integration and the result of the automaton calculations greatly improves, as shown in Fig. 6(b). We note that the automaton [Eq. (11)] gives a very accurate representation of the relaxation. The assumption of the simplified automaton of Eq. (14) that neglects the interactions with the neighbors and assumes complete stress relaxation during a slip event, works very well. 


\section{SUMMARY AND CONCLUSIONS}

In this paper we have studied the stress relaxation in a BK model within three increasingly simplified models, by assuming that the system relaxes from the solitary state. (i) A full continuum calculation for the dynamics and relaxation of a driven BK-model as a reference system. This approach allows us to study the system up to $t \sim 10^{5}$. (ii) An exact automaton [Eq. (11)] for the solitary state, which uses the distribution of the full continuum calculation given in Fig. 4(a) as an initial state, to be able to study the relaxation over many more decades. The agreement between the exact automaton and the full dynamical simulations is very good at all temperatures, also when the actual dynamical model is not in the solitary state [15]. Furthermore, we show that although the stress of three blocks changes due to the slip of only one block, the reduction of the average stress depends only on the force acting on the moving block just before it slips. From the results of this automaton we construct the distribution of forces at the moment a block slips $\left[P_{\text {init }}(\sigma)\right.$, Fig. 5]. (iii) A simplified automaton [Eq. (14)] based on the distribution $P_{\text {init }}(\sigma)$, which we do not solve numerically, but we use as inspiration for an approximate analytical solution. Consequently we have been able to describe the time dependence of the mean of this distribution by an integral that can be calculated analytically for short, intermediate, and long times if we assume that this statistical distribution is uniform. The analytical results in these three regimes are very similar to the one described in Ref. [11] to describe hierarchically constrained systems by means of very different assumptions. Intuitively the stress relaxation of the strongly connected elements of the BK model seems to indicate a stress relaxation that is dominated by the interaction between the elements. In this respect, the BK model can also be viewed as hierarchical constrained because blocks increase the force on their neighbors when they slip, increasing the probability that their neighbors will slip as well. However, the logarithmic relaxation has its origin in the fact that, in the relevant range of parameters, the elements can essentially be described as independent and their stress distribution as uniform.

Our results show that an alternative route to logarithmic relaxation is the occurrence of localized events of individual elements due to a uniform distribution of forces. These results are not against the idea that slow relaxation is characteristic of complex systems, the complexity being cast in the equal likeliness of all values of the forces acting on each block rather than in the detailed interactions with the other elements of the system.

\section{APPENDIX: ANALYTICAL APPROXIMATIONS OF THE STRESS RELAXATION}

By making the substitution as in Ref. [11],

$$
\eta=\nu t \exp \left(-\beta \epsilon\left[1-\sigma^{2}\right]\right), \quad \Rightarrow d \sigma=\frac{1}{2 \beta \epsilon \sigma} \frac{d \eta}{\eta},
$$

we can estimate the behavior at short, intermediate, and long times, by rewriting Eq. (20) as

$$
\bar{\sigma}(t)=\frac{1}{2 \beta \epsilon} \int_{\nu t \exp (-\beta \epsilon)}^{\nu t} \frac{d \eta}{\eta} e^{-\eta} .
$$

For very short times $t \approx 1 / \nu$ the exponential in the integral of Eq. (A2) can be neglected with respect to $1 / \eta$. We can expand the exponential of Eq. (A2) to first order in $\eta$ and integrate to give

$$
\lim _{t \rightarrow 0} \bar{\sigma}(t)=\frac{1}{2}-\frac{1}{2 \beta \epsilon}\left\{[1-\exp (-\beta \epsilon)] \nu t+O\left(t^{2}\right)\right\} .
$$

When $t \gg \exp (\beta \epsilon) / \nu$, i.e., for long times, the exponential on the right-hand side of Eq. (A2) decays much faster than $1 / \eta$. We can roughly approximate Eq. (A2) by

$$
\begin{aligned}
\lim _{t \rightarrow \infty} \bar{\sigma}(t) & \approx \frac{1}{2 \beta \epsilon} \int_{\nu t \exp (-\beta \epsilon)}^{\nu t} \frac{d \eta}{\nu t \exp (-\beta \epsilon)} e^{-\eta} \\
& =\frac{\exp (\beta \epsilon)}{2 \nu t \beta \epsilon}\{\exp [-\nu t \exp (-\beta \epsilon)]-\exp (-\nu t)\} \\
& \approx \frac{\exp (\beta \epsilon)}{2 \beta \epsilon} \frac{\exp [-\nu t \exp (-\beta \epsilon)]}{\nu t} .
\end{aligned}
$$

For very long times $t$ this is proportional to $\exp (-t / \tau)$ with $\tau=\exp (\beta \epsilon) / \nu$.

The solution for intermediate times can be found by integrating Eq. (A2) in parts such that

$$
\begin{aligned}
\bar{\sigma}(t)= & \frac{1}{2 \beta \epsilon}[\log (\eta) \exp (-\eta)]_{\nu t}^{\nu t} \exp (-\beta \epsilon) \\
& +\frac{1}{2 \beta \epsilon} \int_{\nu t \exp (-\beta \epsilon)}^{\nu t} \log (\eta) \exp (-\eta) d \eta .
\end{aligned}
$$

Evaluating the first part of Eq. (A5) gives

$$
\begin{array}{r}
\frac{1}{2 \beta \epsilon}\left[\log (\nu t) e^{-\nu t}-\log \left(\nu t e^{-\beta \epsilon}\right) \exp \left(-\nu t e^{-\beta \epsilon}\right)\right] \\
=\frac{1}{2 \beta \epsilon} \log (\nu t)\left[\exp (-\nu t)-\exp \left(-\nu t e^{-\beta \epsilon}\right)\right] \\
+\frac{1}{2} \exp \left(-\nu t e^{-\beta \epsilon}\right) \approx \frac{1}{2}-\frac{1}{2 \beta \epsilon} \log (\nu t),
\end{array}
$$

where the approximation is valid when $\exp (-\nu t) \approx 0$ and $\exp \left(-\nu t e^{-\beta \epsilon}\right) \approx 1$.

For $1 / \nu \ll t \ll \exp (\beta \epsilon) / \nu$, the last term of Eq. (A5) can be approximated as

$$
\begin{aligned}
& \frac{1}{2 \beta \epsilon} \int_{\nu t \exp (-\beta \epsilon)}^{\nu t} \log (\eta) \exp (-\eta) d \eta \\
& \quad \approx \frac{1}{2 \beta \epsilon} \int_{0}^{\infty} \log (\eta) \exp (-\eta) d \eta=-\frac{\hat{\gamma}}{2 \beta \epsilon}
\end{aligned}
$$

with $\hat{\gamma}$ the Euler-Mascheroni constant $\hat{\gamma} \approx 0.577$. Summing Eqs. (A6) and (A7) results in the most important result of the Appendix,

$$
\bar{\sigma}(t) \approx \frac{1}{2}-\frac{1}{2 \beta \epsilon}[\hat{\gamma}+\log (\nu t)]
$$


[1] K. Binder and A. P. Young, Rev. Mod. Phys. 58, 801 (1986).

[2] M. D. Ediger, C. A. Angell, and S. R. Nagel, J. Phys. Chem. 100, 13200 (1996).

[3] J. A. TenCate, E. Smith, and R. A. Guyer, Phys. Rev. Lett. 85, 1020 (2000).

[4] L. Bocquet, E. Charlaix, S. Ciliberto, and J. Crassous, Nature (London) 396, 735 (1998).

[5] J. D. Bryngelson, J. N. Onuchic, N. D. Socci, and P. G. Wolynes, Proteins: Struct., Funct., Genet. 21, 167 (1995).

[6] M. Cloitre, R. Borrega, and L. Leibler, Phys. Rev. Lett. 85, 4819 (2000).

[7] O. B. Tsiok, V. V. Brazhkin, A. G. Lyapin, and L. G. Khvostantsev, Phys. Rev. Lett. 80, 999 (1998).

[8] K. Büntemeyer, H. Lüthen, and M. Böttger, Planta 204, 515 (1998).

[9] J. Nieuwland, R. Feron, B. A. H. Huisman, A. Fasolino, C. W. Hilbers, J. Derksen, and C. Mariani, Plant Cell 17, 2009
(2005).

[10] R. G. Palmer, D. L. Stein, E. Abrahams, and P. W. Anderson, Phys. Rev. Lett. 53, 958 (1984).

[11] J. J. Brey and A. Prados, Phys. Rev. E 63, 021108 (2001).

[12] F. R. N. Nabarro, Mater. Sci. Eng., A 309-310, 227 (2001).

[13] R. Burridge and L. Knopoff, Bull. Seismol. Soc. Am. 57, 341 (1967).

[14] B. N. J. Persson, Phys. Rev. B 51, 13568 (1995).

[15] B. A. H. Huisman and A. Fasolino, Phys. Rev. E 72, 016107 (2005).

[16] B. N. J. Persson, Phys. Rev. B 48, 18140 (1993).

[17] F. P. Bowden and D. Tabor, The Friction and Lubrication of Solids (Oxford University Press, Oxford, 2001).

[18] For illustrational purposes we use a rather high temperature so that the relaxation of the stress after the plate has stopped is evident on a linear time scale. 